\title{
Exergy Analysis for Brayton and Inverse Brayton Cycles with Steam Injection
}

Betelmal EH*, Farhat S and Agnew B

Faculty of Engineering, Department of Mechanical Engineering, Tripoli University, Tripoli, Libya

\begin{abstract}
Global economic and social development has led to the growth in demand for electrical power. Gas turbines play an essential role in many societies, and as the requirements for power generation increase, the power output and thermal efficiency of gas turbines must also increase. This paper presents one of the ways to improve the performance of the gas turbine with a study of exergy analysis of combined Brayton and inverse Brayton cycles with steam injected into the combustion chamber. The effect of variation of operating conditions (compression ratio and ambient temperature) on the performance of gas turbine (thermal efficiency, power) were investigated, the results were compared with the same cycle, but without injection with the analytical formulae of exergy and exergy destruction. The programming of performance model for gas turbine was developed utilizing the commercially available software IPSEpro. The analysis shows that the highest exergy destruction occurs in the combustion chamber. In addition, the performance has been improved by using steam injection by $11 \%$ in efficiency and $57 \%$ in power output and decrease linearly with the increase of the ambient temperature. However, steam injection increases the specific fuel consumption and heat rate. Thus, the thermodynamic parameters on cycle performance are economically feasible and beneficial for the gas turbine operations.
\end{abstract}

Keywords: Gas turbine; Brayton cycle; Inverse brayton cycle; Exergy analysis; Exergy loss

\section{Introduction}

Under the Kyoto Protocol, the world's wealthier countries assumed binding commitments to reduce greenhouse gas emissions [1] and the energy supply to the demand limited day after day around the world. The growing demand of the power has made the power plants of scientific interest, but most of the power plants are designed by the energetic performance criteria based on the first law of thermodynamics only. The real beneficial energy loss cannot be determined by the first law of thermodynamics, because the real plant inefficiencies are not related to energy loss but to exergy destruction. Exergy analysis and determining the exergy destruction of each component of the power plant is a useful concept in ecology, and sustainability because it can be used as a common measure of research quality along with quantity, and to improve system efficiency. In the recent years, the various methods have been tested for improving the gas turbines performance. Among several methods shown their success in increasing the performances, the steam injected gas turbine cycle consists of introducing a high amount of steam at various points in the cycle. For several years, the injection of steam in the combustion chamber has represented a common way to improve the performance of gas turbine power plants, increasing both the power output and the efficiency and reducing, at the same time, $\mathrm{NO}_{\mathrm{x}}$ emissions $[2,3]$.

In this paper, the performance characteristics of Brayton and inverse Brayton cycles with steam injected into combustion chamber are analysed and it is compared with the performances of the Brayton and the inverse Brayton cycle.

\section{System Description}

The system as shown in Figure 1 is constructed from a top cycle (Brayton cycle) and a bottoming cycle (inverse Brayton cycle). The purpose of the compressor is to compress the inlet air (raises its pressure). Fuel is mixed with the high-pressure air and burnt in special chambers called combustors. The hot pressurized gas exits the combustors at a very high temperature (upto $1100^{\circ} \mathrm{C}$ ). This gas

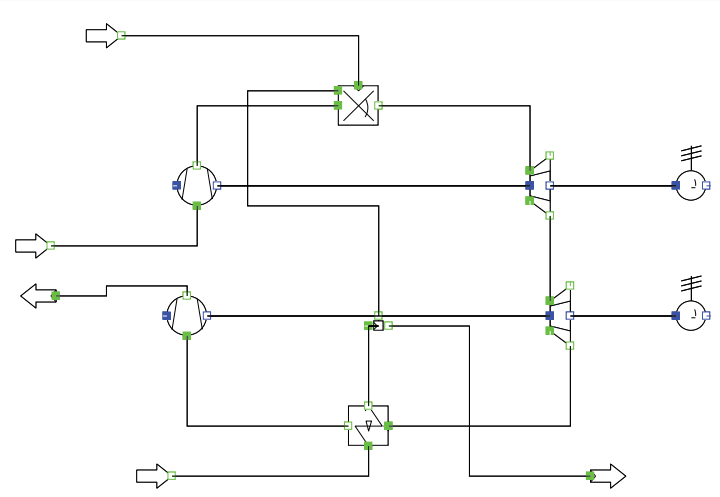

Figure 1: Brayton and inverse Brayton cycles with steam injection.

then passes through a gas turbine, giving the turbine energy to spin the compressor and the power for a generator to produce electricity. Because some of its heat and pressure energy has been transferred to the turbine, the gas leaves the power turbine at a lower temperature and pressure rather than discharged to the atmosphere, but it is directed to an inverse gas turbine, called an inverse Bryton cycle. Hot gasses are expanded through a second turbine to below ambient pressure, and then compressed back to ambient pressure using works from this turbine. Steam generated from the heat recovery is injected into the

*Corresponding author: Betelmal EH, Faculty of Engineering, Department of Mechanical Engineering, Tripoli University, Tripoli, Libya, Tel: +218 21-4627901; E-mail: e.betelmal@hotmail.com

Received September 30, 2017; Accepted October 30, 2017; Published November 06, 2017

Citation: Betelmal EH, Farhat S, Agnew B (2017) Exergy Analysis for Brayton and Inverse Brayton Cycles with Steam Injection. J Appl Mech Eng 6: 292. doi: $10.4172 / 2168-9873.1000292$

Copyright: (c) 2017 Betelmal EH, et al. This is an open-access article distributed under the terms of the Creative Commons Attribution License, which permits unrestricted use, distribution, and reproduction in any medium, provided the original author and source are credited. 
combustion chamber of a gas turbine, increasing the flow through the turbine. The energy recovered by the turbine can be increased by lowering the exhaust temperature. Result is lower exhaust temperature, higher performance. The IPSEpro program has been used to model and examine the combined thermodynamic cycles to determine the optimum mode of operation and configuration [4]. This work examines the performance of a combined Brayton and inverse Brayton cycles according to the second law of thermodynamics using the exergy analysis in the operation of the combined cycle.

\section{Thermodynamic Analysis}

The present study introduces a comparative energy and exergy analysis for Brayton and inverse Brayton cycles with and without Steam Injection. The analysis investigated the effects of different ambient temperatures. The IPSEpro program will be used to model and examine the combined thermodynamic cycles to determine the optimum mode of operation and configuration. The steam entering the combustor is at superheated condition, being an energy carrier of recovered heat from the hot exhaust and it is assumed not to participate in the chemical reactions of the combustion. The pressure of the steam when injected must be somewhat higher than the pressure of air coming from the combustor. To keep a constant turbine inlet temperature, the amount of fuel supplied in the combustor is larger for a steam injection gas turbine compared to a common gas turbine. The increase of fuel power in a steam injection gas turbine will thus be smaller than the gain in net power output of the gas turbine, which gives that higher efficiency. By injecting steam in the combustion zone, the peaks in flame temperature are decreased. Usually, the maximum steam injection rate is about $15 \%$ to $20 \%$ of the air mass flow $[5,6]$. The steam, which is injected in the combustion chamber of the gas turbine, is blended with the combustion gases, and will follow the gas steam to the stack.

\section{The Input Data and Some Assumptions}

Methane $\left(\mathrm{CH}_{4}\right)$ gas enters a steady-flow adiabatic combustion chamber at $25^{\circ} \mathrm{C}$ and 25 bar.

Mass of air $\mathrm{m}_{\text {air }}=30 \mathrm{~kg} / \mathrm{s}$

Inlet temperature $\mathrm{T}_{\text {air }}=10^{\circ} \mathrm{C}$

Inlet pressure $\mathrm{P}_{\text {air }}=1 \mathrm{bar}$

\section{The input data for compressor}

Compressor isentropic efficiency $\eta_{s}=0.85$

Compressor mechanical efficiency $\eta_{\mathrm{m}}=0.98$

Pressure ratio $\mathrm{P}_{2 /} \mathrm{P}_{1}=14$

Pressure drop in the combustion chamber $=0.1 \mathrm{bar}$

\section{The input data for turbine}

Turbine mechanical efficiency $\eta_{\mathrm{m}}=0.99$

Turbine isentropic efficiency $\eta_{s}=0.9$

Turbine inlet exhaust gas temperature $\mathrm{T}_{\max }=1100^{\circ} \mathrm{C}$

The characteristics of heat exchanger are

Pressure drop of the hot temperature side $=0.1 \mathrm{bar}$

Pressure drop of the low temperature side $=0.1$ bar

The temperature increase of the air during the compression is:

$$
\left(\frac{d T_{1-2}}{T_{1}}\right)_{\text {actual }}=\left(\left(\frac{P_{2}}{P_{1}}\right)^{\frac{\gamma-1}{\gamma}}-1\right) / \eta_{s}
$$

The pressure ratio $P_{3} / P_{2}$ can be obtained directly from the combustion chamber pressure loss

$$
\frac{P_{3}}{P_{2}}=1-\left[\Delta P_{b} / P_{2}\right]
$$

The overall efficiency of the gas turbine and inverse gas turbine cycle is calculated using the following equation 1

$$
\eta_{C C}=\frac{P_{G T}+P_{I G T}}{Q_{i n}}
$$

Where:

$P_{G T}$ is the power of the gas turbine.

$P_{I G T}$ is the power of the inverse gas turbine cycle.

$Q_{i n}$ is the input energy of the gas turbine.

The steam injected is not taking active part in the combustion process, but will be heated to the same temperature as the rest of the gas, i.e. to the turbine inlet temperature. Since the steam is not reacting in the combustion process, the gas and steam flows can be treated separately. The heat balance thus becomes:

$$
m_{\text {air }} \cdot h_{2, \text { air }}+m_{f} \cdot L H V+m_{S t} \cdot h_{S t, i n}=\left(m_{3, \text { air }}+m_{f}\right) \cdot h_{3, \text { gas }}+m_{S t} \cdot h_{S t, e x}
$$

The mass fuel air ratio $(\beta)$ is defined identically as for the common gas turbine:

$$
\begin{aligned}
& \beta=\frac{m_{f}}{m_{\text {air }}} \\
& \beta=\frac{h_{3, \text { gas }}-h_{2, \text { air }}+\frac{m_{S t}}{m_{\text {air }}}\left(h_{S t, e x}-h_{S t, \text { in }}\right)}{L H V-h_{3, \text { gas }}}
\end{aligned}
$$

\section{Exergy Destruction in Different Components of Com- bined Cycle}

The complex thermodynamic analysis of combined cycle has been based on the second law of thermodynamics, because the conventional first law analysis of any thermodynamic system has the capability to determine the energy distribution across the system boundaries but does not explain the means of energy degradation that cause decreases in the power output of the system. The exergy analyses for combined cycle components have been conducted to determine the energy loss caused by irreversible processes.

Exergy analysis usually predicts the thermodynamic performance of an energy system and the efficiency of the system components by quantifying the entropy generation of the components. This analysis provides a tool for the optimal design and operation of complex thermal systems. The exergy and irreversibility equations for each component are written as follows [5]:

\section{Compressor}

The exergy and irreversibility in compressor is given by

$$
\begin{aligned}
& \Delta \varphi=m_{a}\left(h_{1}-h_{2}\right)-T_{o} m_{a}\left(s_{1}-s_{2}\right) \\
& I_{c}=m_{a} T_{0}\left(\mathrm{~S}_{2}-\mathrm{S}_{1}\right)
\end{aligned}
$$

Where 


$$
S_{2}-s_{1}=c_{p_{a}} \ln \frac{T_{2}}{T_{1}}-R_{a} \ln \frac{P_{2}}{p_{1}} \text { and } R_{a}=C_{P a} \frac{(\gamma-1)}{6}
$$

\section{Combustion chamber}

As an approximation, the virtual power is used to calculate the exergy of the combustor component. The exergy balance of this component and exergy loss due to irreversibility in the combustion chamber is given by

$$
\begin{aligned}
& \varphi_{c . c}=Q_{i n}+T_{o} S_{g e n} \\
& I_{c \cdot c}=T_{0} S_{g e n}
\end{aligned}
$$

Where,

$S_{g e n}=\left(\dot{m}_{a}+\dot{m}_{f}\right)\left(c_{p} \ln \frac{T_{3}}{T_{2}}\right)-\frac{\dot{Q}_{i n}}{T_{a v}}$

And $T_{a v}=$ average $\left(\mathrm{T}_{3}, \mathrm{~T}_{2}\right)$

\section{Turbines}

The exergy loss due to irreversibility in gas turbine is given by

$$
\begin{aligned}
& \varphi_{T}=m_{a}\left(h_{3}-h_{4}\right)-T_{o} m_{a}\left(s_{3}-s_{4}\right) \\
& I_{G T}=m_{g} T_{o}\left(s_{4}-s_{3}\right)
\end{aligned}
$$

Where

$$
\left(s_{4}-s_{3}\right)=C_{P g} \ln \frac{T_{4}}{T_{3}}-R_{g} \ln \frac{P_{4}}{P_{3}}
$$

And

$R_{g}=C_{P g} \frac{(\gamma-1)}{\gamma}$

\section{Heat exchanger}

The exergy loss due to irreversibility in heat exchanger is given by

$$
\begin{aligned}
& \Delta s_{\text {hot }}=s_{\text {hot-ex }}-s_{\text {hot-in }} \\
& \Delta s_{\text {hot }}=s_{\text {hot-ex }}-s_{\text {hot-in }} \\
& \varphi_{\text {cold }}=\left(h_{\text {cold-ex }}-h_{\text {cold-in }}\right)-T_{o}\left(s_{\text {cold-ex }}-s_{\text {cold }- \text { in }}\right) \\
& \varphi_{\text {hot }}=\left(h_{\text {hot-in }}-h_{\text {hot-ex }}\right)-T_{o}\left(s_{\text {hot-in }}-s_{\text {hot-ex }}\right) \\
& I_{E X}=T_{o} \Delta \dot{S}_{o}=T_{o}\left[m_{\text {hot }}\left(s_{4}-s_{6}\right)-m_{\text {cold }}\left(s_{\text {ex }}-s_{\text {in }}\right)\right]
\end{aligned}
$$

Where,

$$
s_{6}-s_{4}=C_{P g} \ln \frac{T_{6}}{T_{4}}-R_{g} \ln \frac{P_{6}}{P_{4}}
$$

\section{Results and Discussion}

Based upon the methodology developed and the thermodynamic equations shown, the effect of ambient temperature on the performance and on the destruction of exergy due to irreversibility in the various components of the gas turbine and inverse gas turbine, with and without steam injection is displayed graphically.

The following figures show the change the performance of the gas turbine and inverse gas turbine cycle with changing ambient temperature and compression ratio. Exergy destruction is obtained

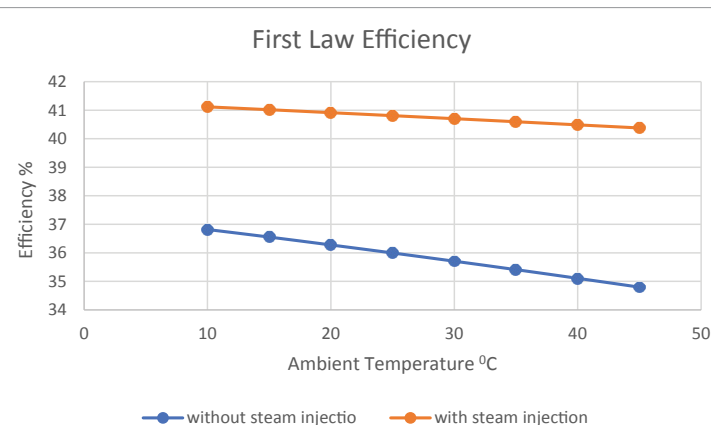

Figure 2: Thermal gas turbine efficiency as a function of ambient temperature with and without steam injection.

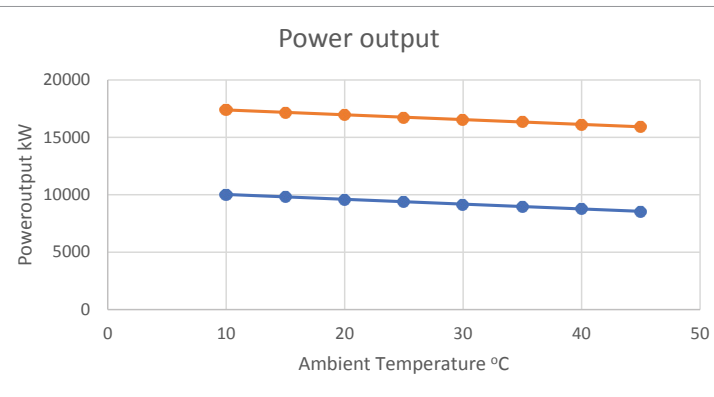

Figure 3: The gas turbine power output as a function of ambient temperature.

Fuel Consumption

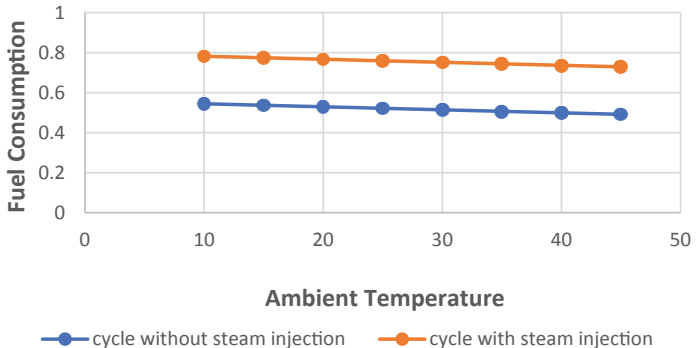

Figure 4: The gas turbine fuel consumption as a function of ambient temperature.

from the exergy (second law) analysis. The exhaust gases of the turbine have an elevated temperature and therefore significant exergy, hence ensuring high thermodynamic efficiency of the combined cycle.

Figure 2 shows the combined cycle efficiency, decreasing because of increasing ambient temperature. The power output has been affected by ambient temperature as shown in Figure 3 and clearly shows that the power output decreases as temperature increases. These figures show a significant increase in the performance with the use of steam injection. Combine cycle efficiency more than $40 \%$ can be achieved with this system when injecting steam into the combustion chamber of a gas turbine, whereas it drops when use gas turbine without steam injection, combined gas turbine and inverse gas turbine cycle represents an attractive option for power units, especially in the modern world, where the number of successfully operated base load combined cycle lastingly increasing and better efficiency. The generated power and efficiency of gas turbine plants depend on the temperature of the inlet air.

The following figure shows the increasing in the fuel consumption 
with stem injection and slightly decreases with ambient temperature increases (Figure 4).

It can be seen in Figure 5 the effect of ambient temperature on the exergy destruction rate of the system component at full load condition

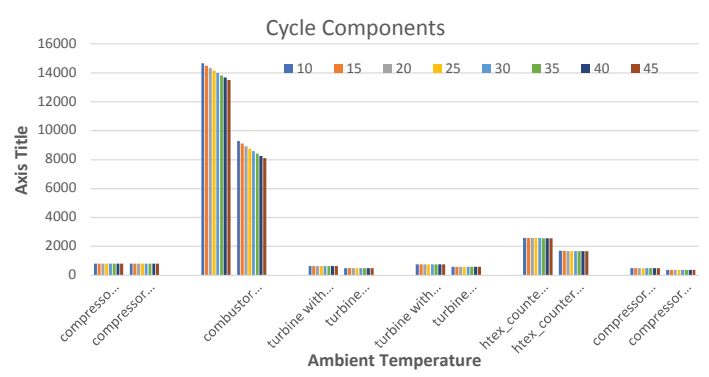

Figure 5: The exergy destruction of the combined cycle with and without injection as a function of ambient temperature.

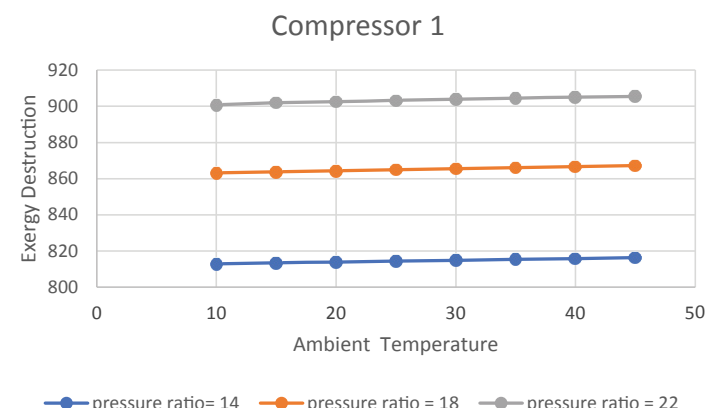

Figure 6: Exergy destruction of the compressor with ambient temperature at different pressure ratio.

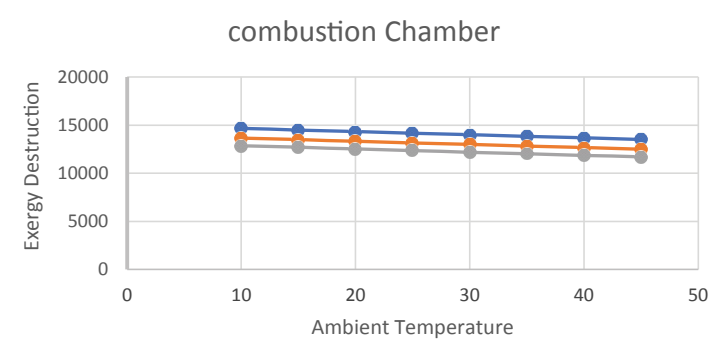

$\longrightarrow$ presure ratio $=14 \longrightarrow$ pressure ratio $=18 \rightarrow$ pessure ratio $=22$

Figure 7: Exergy destruction of the combustion chamber with ambient temperature at different pressure ratio.

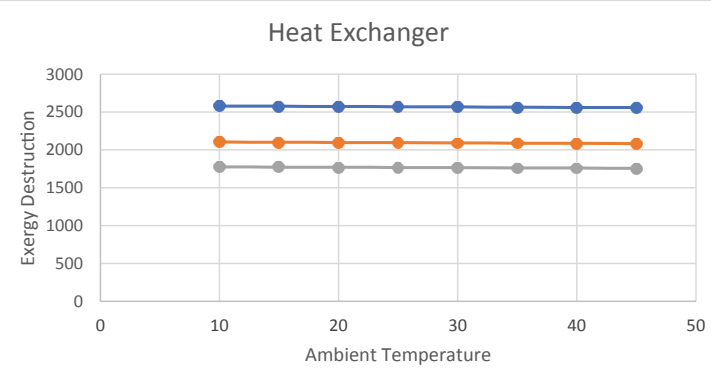

Figure 8: Exergy destruction of the heat exchanger with ambient temperature at different pressure ratio.

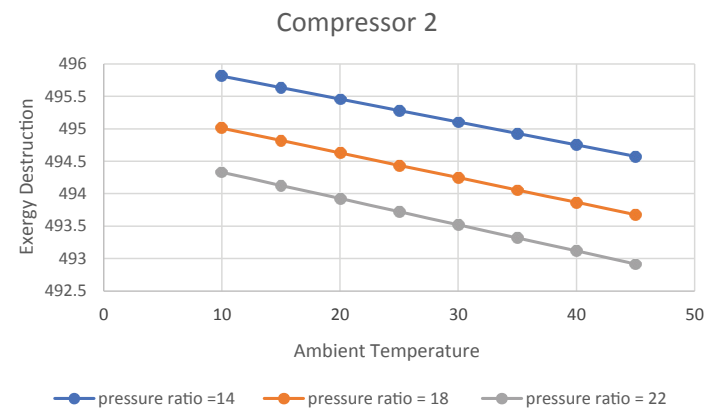

Figure 9: Exergy destruction of the inverse gas turbine compressor with ambient temperature at different pressure ratio.

in both cases (with and without steam injection). With an increase in ambient temperature from $10^{\circ} \mathrm{C}$ to $45^{\circ} \mathrm{C}$ the exergy destruction rate of a compressor is slightly higher as the ambient temperature increases and doesn't change with steam injection, whereas it slightly decreases in other system components. The maximum exergy destruction takes place in the combustion chamber followed by gas turbine and as shown in this figure, more exergy destruction when using steam injection.

Figure 6 show the exergy loss in the compressor with variation in the compressor pressure ratio and ambient temperature. There is a clear effect of the compressor pressure ratio of exergy loss in compressor. As high compressor pressure ratio of 22 the exergy loss in the compressor is higher and as the compressor pressure decreases to 14, the exergy loss is minimizing. At any condition as the compressor pressure ratio increases, the exergy loss increases.

Figures 7-9 show the variation of exergy loss in the combustion chamber, heat exchanger and compressor of inverse gas turbine with variation in the compressor pressure ratio and ambient temperature. At high pressure ratio of 22 , the exergy loss in the combustion chamber is lower and as the pressure ratio decreases, the exergy loss increases. There is similar behaviour in exergy loss that can be seen in the heat exchanger and the compressor of the inverse gas turbine as the compressor pressure ratio and ambient temperature increase, the exergy loss decreases. In combustion chamber 35\% to 50\% of exergy loss is calculated.

Figures 10 and 11 show the variation of exergy loss in gas turbine with variation in the compressor pressure ratio and ambient temperature. At a high compression ratio of 22, the exergy loss in the gas turbine is higher and as the pressure ratio decreases to 14 the exergy loss is minimized. There is similar pattern in exergy loss that can be seen in inverse turbine. At any compression ratio, as the ambient temperature increases, the exergy loss decreases. In gas turbine $2 \%$ to $7 \%$ of exergy loss is obtained.

\section{Conclusion}

The simulation program IPSEpro has been applied successfully to the gas turbine cycle. Energy and exergy analysis based on the thermal efficiency, exergy was analysed. The production of electricity and efficiency are highest at cooler times of the day and the reverse at the hottest time of the day when they are needed most. Steam generated from heat recovery is injected into the combustion chamber of a gas turbine, increasing the flow rate through the turbine, this causes an increase the power output of a gas turbine and the electrical efficiency, thus that the power output of the gas turbine with steam injection will be higher than the common gas turbine. A steam-injected gas turbine can reach an electrical efficiency up to $40 \%$. There are limitations of 


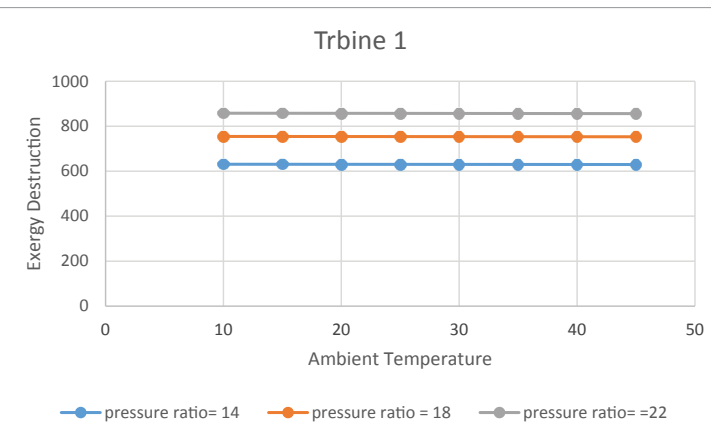

Figure 10: Exergy destruction of the turbine with ambient temperature at different pressure ratio.

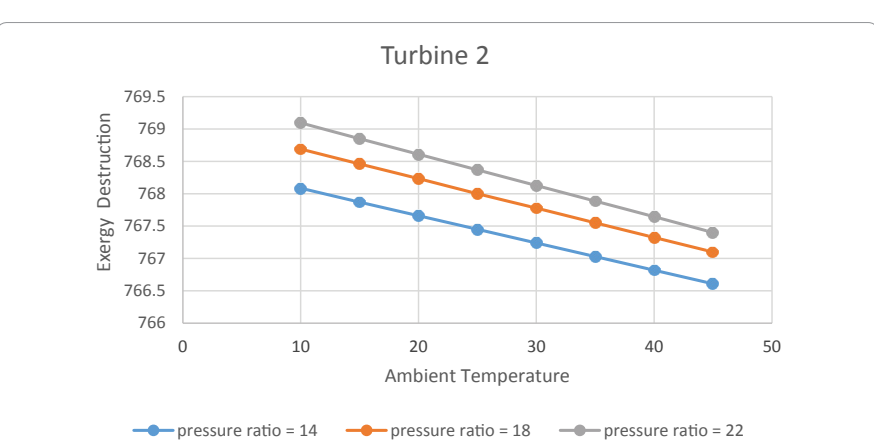

Figure 11: Exergy destruction of the inverse gas turbine with ambient temperature at different pressure ratio. the amount of steam that can be injected, based on the above analysis; the following conclusions are made by varying the pressure ratio of the compressor and ambient temperature,

- The exergy analysis gives a real picture about the losses which occurred in the different plant components.

- More exergy losses occur in the combustion chamber due to combustion irreversibility and this must be reduced with the aid of advanced in the modern technology as the combustion process is necessary.

- There are clear effects in the exergy losses when changing pressure ratio. At higher pressure ratio, the exergy losses in the components, compressor, turbine and inverse turbine are higher.

- The optimum turbine inlet temperature and pressure ratio should be the next focus of study for minimizing the total exergy losses in all the components.

\section{References}

1. Mustfa B, Reilly JM, Jacoby HD (2000) The Kyoto Protocol and developing countries. Energy Policy 28: 525-536.

2. Bathie WW (1996) Fundamentals of gas turbines. (2nd edn), John Wiley \& Sons, Inc, NY, USA.

3. Jonssona M, Yan J (2005) Humidified gas turbines: A review of proposed and implemented cycles. Energy 30: 1013-1078.

4. Simulation Technology (2001) IPSE pro process simulator, Manual version.

5. Sonntag R, Wylen VG (1991) Introduction to thermodynamics classical and statistical. John Wiley \& Sons, NY, USA.

6. Erlich C (1999) Steam injection gas turbine cycle. p: 1-6. 